\title{
Consistency and Comparison of Monomodal Multi-Temporal Medical Image Registration- Segmentation and Mathematical Model for Glioblastoma Volume Progression
}

\author{
E. IRMAK
}

\begin{abstract}
Tumor volume progression analysis and tumor volume measurement are very common tasks in cancer research and image processing fields. Tumor volume measurement can be carried out in two ways. The first way is to use different mathematical formulas and the second way is to use image registration method. In this paper, using 3D medical image registration-segmentation algorithm, multiple scans of MR images of a patient who has brain tumor are registered with different MR images of the same patient acquired at a different time so that growth of the tumor inside the patient's brain can be investigated. Tumor volume progression analysis and tumor volume measurement are performed using image registration technique and the results are compared with the results of tumor volume measurement by mathematical formulas. For the first patient, grown brain tumor volume is found to be $10345 \mathrm{~mm}^{3}$, diminished brain tumor volume is found to be $15278 \mathrm{~mm}^{3}$ and unchanged brain tumor volume is found to be $20876 \mathrm{~mm}^{3}$. Numerical results obtained by image registration model proves that medical imageregistration method is not only between the true ranges but also is very close to the best mathematical formula. Medical image registration-segmentation are implemented to 19 patients and satisfactory results are obtained The results are compared with the results obtained from mathematical methods. An advantageous point of medical image registration-segmentation method over mathematical models for brain tumor investigation is that grown, diminished, and unchanged brain tumor parts of the patients are investigated and computed on an individual basis in a threedimensional (3D) manner within the time.
\end{abstract}

Index Terms - Brain tumor growth, Medical image registration, Medical image segmentation, Tumor volume computing.

\section{INTRODUCTION}

\section{A. Brain Tumors, Imaging and Importance}

B RAIN TUMORS have been announced as one of the most fatal cancers in the western population [1].

EMRAH IRMAK, is with Department of Electrical and Electronics Engineering University of Alanya Alaaddin Keykubat University, Antalya, Turkey, (e-mail: emrah.irmak@alanya.edu.tr)

iD https://orcid.org/0000-0002-7981-2305

Manuscript received May 06, 2020; accepted August 11, 2020.

DOI: $\underline{10.17694 / \text { bajece. } 733330}$
Moreover, Kohler et al. [2] declared that probability of occurrence of primary tumors of the nervous system or brain is 25 per 100,000 . By almost a third are malignant and the remaining are benignant or some kind of benignant [3]. World Health Organization (WHO) has introduced a grading scheme which categorizes brain tumors between I and IV. Glioblastoma (WHO grade IV) is known as the most fatal and the most frequent brain tumor which shows very rapid growth [4]. Although treatment methods such as surgery, radiation, chemotherapy are available for treatment of glioblastoma average survival time is 15 months because of the infiltrating nature of glioblastoma [5]. That is why the special care should be given to treatment of glioblastoma. Actually treatment of glioblastoma becomes one of the most challenging fields in oncology [4]. There is a thriving attention and application of glioblastoma progression analysis in clinical diagnostics and analysis. Various researchers have showed that MRI is superior to CT for diagnostic brain imaging [6-9]. Magnetic resonance imaging is a standard and non-invasive technique. The readers who are interested in image processing using glioblastoma MRI images can examine the paper by K. Kaplan et al. [10]. MRI is widely available in clinics. Consequently, MRI in combination with other imaging modalities based studies is more feasible and rational in a clinical point of view. Nevertheless, it should be kept in mind that for a final decision and diagnosis, biopsy and histology are necessary despite the all suitability and practicability of non-invasive imagings. Thanks to the big amount of data, the principal focus of this paper will be on MRI and glioblastoma tumor volume calculation.

It is quite obvious that glioblastoma is a notably deadly disease today and even today's treatment modalities are all around insufficient in curing or even controlling. Glioblastomas are comparatively resistant to $\mathrm{X}$ radiation in comparison with other tumor types. Scientists have introduced that most brain tumor recurrence are located within the primary tumor area [11-13]. Although there are studies on measuring the volume of the brain tumor, the definition of tumor volume is still based on time consuming, highly subjective manual outlining by radiologists, clinicians etc. [3]. Today's treatments for glioblastoma ordinarily need tumor removal using surgical 
methods followed by irradiation of the tumor source.

\section{B. Literature Survey}

Various researchers have studied tumor volume investigation by both measuring and growth analysis [14-19]. Tumor volume measurement is done by a lot of techniques such as 3D I-scan, ultrasonic 3D scanning system, correlation, diameter, height, area calculation etc. These techniques can be mainly categorized into two groups; medical image processing based models and mathematical models [15, 16].

For mathematical models, tumor volume calculation was achieved by using various mathematical formulas [14, 17, 18]. Guthoff [20], for example, made use of area of sphere phenomena, however the usage of that methods were not sustainable now that it was found to be too complicated. Char et al. [14] searched growth rate using an exponential growth model with tumor volume formula. Difference in tumor size with respect to time was considered as growth rate. Li et al. [17] considered the tumor volume as a part of spheroid intersected by a sphere with a very detailed formula. Many researchers believed the change in tumor diameter to be model for the whole brain tumor volume [21-23]. These researchers found it enough to measure only one dimension of the tumor. On the other hand, some researchers measured average of two dimensions [24, 25]. Others considered the volume to be proportional to area and measured tumor area from measurements of two perpendicular diameters. E. Richtig et al. [18] investigated that tumor volume, calculated by the easy to use formula of the half volume of a rotation ellipsoid, rotated around the $y$-axis, is a better than tumor diameter or tumor height.

For medical image processing based models, a wide range of medical image techniques have been presented with the developments in medical image processing field over the years. As these techniques were independently studied, a large body of research is evolved. As far as it goes there is a wide range of techniques. However, now that every method is designed for a specific application rather than specific types of problems, categorizations and comparison of techniques with each other become difficult. Fortunately, measurement was made using medical image segmentation, medical image registration and the combination of segmentation and registration. Medical image registration with segmentation is very important for monitoring glioblastomas growth during therapy as well as glioblastoma tumor volume measurement. Since the year of 2000, a growing interest and application of medical image processing can be seen from the large number of scientific papers [26-29]. See Figure 1 for frequency of publications in medical image registration field between years 2000 to 2014 . Brock et al. [30] used a deformable registration method for tumor registration. The drawback was the substantial processing time. Kaus et al. [31] explored a surface-based registration technique and implemented on human brain. The author achieved a processing time of a few seconds however manually selecting control point was quite complicated and tedious. Maxwell's demons registration was used with lesion growth model by Cuadra et al. [32]. Bloch et al. [33] applied morphology operators to brain diagnosis. They made use of fuzzy set framework for brain MR images and showed several methods of registration of information. Wavelet based methods are getting increase in medical image registration for brain tumor analysis as well. Quite a lot of studies can be found about registration of medical images using wavelet methods [34-39]. Neural network methods are also very popular in medical image registration field to investigate brain tumor volume analysis using MR images with brain tumor. For example, Ozyurt et al. [28] proposed deep convolutional neural network model to detect glioblastoma (malignant tumor) from brain MRI images. Pohl et al. [40] is a good source about the registration of medical images which have slowly evolving brain tumors. They presented a registration technique that includes registration and segmentation together. Bauer et al. [41] prepared a good survey about medical image registration techniques for the brain tumor volume investigation using MR images. Angelini et al. [42] used affine registration to compute and compare intensity difference maps directly for tumor growth.

\section{Motivation}

The motivation of this research paper is to design computer aided diagnosis (CAD) system for brain tumor volume measurement and brain tumor screening. Today, brain tumor volume is generally measured by measuring the length and width of the brain using a caliper which is time consuming and prone to manual errors. Although measuring length and width of brain tumor is possible, height measuring is quite problematic because when measuring tumor height, there mainly exists inaccuracy which causes the largest error to volume results. The difficulty is determination where to position the caliper for measuring a precise height measurement. The proposed method is fully automatic by using the software techniques. That is why the proposed method can solve the mentioned problems regarding to measuring the brain tumor volume successfully with the minimum number of errors. There are also other image processing methods for brain tumor volume measurement. The proposed method is superior to other methods because it does not only measure the tumor volume but also growing, diminishing and unchanged tumor parts.

The rest of this paper is organized as follows. Introduction to brain tumors and brain tumor volume investigation, calculation (Section 1), Materials and Methods for brain tumor investigation and calculation (Section 2), Experimental Results using various methods with comparison to medical image registration method (Section 3), Conclusion (Section 4).

\section{MATERIALS AND METHODS}

\section{A. Mathematical Models for Tumor Volume Progression Analysis}

For tumor volume evaluation, there exist important mathematical models. Various studies show that three dimensional fundamental shape of brain tumor is hemi-ellipsoid [43]. Three dimensions of the tumor measurement are necessary for tumor volume calculations. These are: length $(L)$, width $(W)$, 
height $(H)$. Measurement of tumor volume is a very common task in brain cancer research.

Conventional ellipsoid volume is known as;

$\mathrm{V}=\frac{\pi}{6} *$ (length) $*($ width $) *($ height $)$

Although measuring length and width of brain tumor is possible, height measuring is quite problematic. Because when measuring tumor height, there mainly exists inaccuracy which causes the largest error to volume results. The difficulty is determination where to position the caliper for measuring a precise height measurement [44]. That is why some authors have reduced essential number of dimensions in order to measure tumor volume. John P. Feldman et al. [44] explored a new mathematical method for tumor measurement which uses just two dimensions; length and width. There are other researchers who use two or even one dimension for measuring brain tumor. Table-I shows a lot of mathematical formulas which have been used for tumor volume calculation up to now.

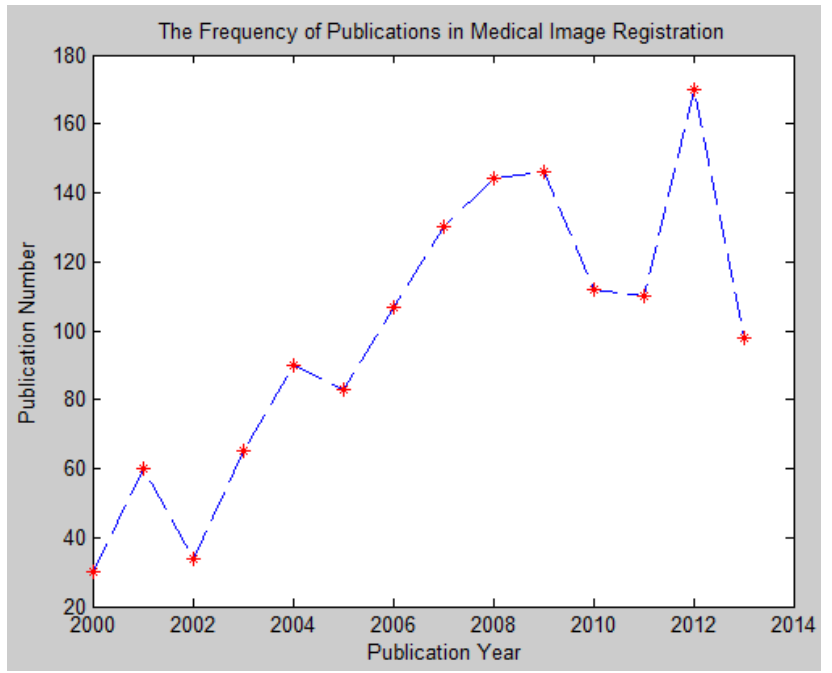

Fig.1. Frequency of papers in Medical Image Registration obtained via ISI

M. M. Tomayko and C.P. Reynolds [45] showed that tumor volume calculation using three-dimensional formula results in the most accurate tumor volume. All the tumor volume measurement formulas are reasonable good at estimating brain tumor but the formula $\pi / 6^{*}($ length $) *($ width $) *($ height $)$ stood out as the best.

\section{B. Medical Image Registration-Segmentation Based Models}

Image registration is a leading-edge for image processing and biomedical engineering fields. Therefore, accurate alignment of the useful information from two or more images is very useful for clinical purposes. Besides, preoperative and intraoperative medical image registration is a critical process for imageguided therapy.

To summarize registration process, Figure 2 is an ideal illustration of how process works. Image which is not changed during registration is called fixed image, the image which is changed, i.e. transformed during registration is called moving image. The purpose of a similarity metric is to return a value indicating how well two images match [46]. Role of optimizer is to define search strategy for the process. Interpolator takes pixel intensities to the new coordinate system according to the geometric transformation that has been found. Interpolator measures the value of intensity difference between the images in the new positions.

TABLE I

TUMOR VOLUME MEASUREMENT FORMULAS

\begin{tabular}{|c|c|c|}
\hline Formula Used & Volume Type & Assumption \\
\hline$\frac{\pi}{6} * L * \boldsymbol{W} * \boldsymbol{H}$ & Ellipsoid & $\begin{array}{c}3 \text { Dimensions are } \\
\text { proportional wrt } \\
\text { tumor growth }\end{array}$ \\
\hline$\frac{\boldsymbol{\pi}}{\mathbf{6}} * \boldsymbol{L} * \boldsymbol{W}^{2}$ & Ellipsoid & $\mathrm{H}=\mathrm{W}$ \\
\hline$\frac{\pi}{6} *\left[\frac{L * W}{2}\right]^{3}$ & Ellipsoid & $\mathrm{H}=\frac{\mathrm{L}^{2} * \mathrm{~W}^{2}}{8}$ \\
\hline$\frac{\pi}{6} *(L * W)^{\frac{3}{2}}$ & Ellipsoid & $\mathrm{H}=\sqrt{L * W}$ \\
\hline $0.4^{*} L * W^{2}$ & Spheroid & $\mathrm{H}=\frac{\mathrm{L}^{2} * \mathrm{~W}^{2}}{\pi}$ \\
\hline$\frac{\mathbf{4}}{3} * \pi *\left(\frac{L+W}{2}\right)^{3}$ & Spheroid & $\mathrm{r}=\frac{L+W}{2}$ \\
\hline$\frac{4}{3} * \pi *\left(\frac{L}{2}\right)^{3}$ & Spheroid & $\mathrm{r}=\frac{L}{2}$ \\
\hline $\boldsymbol{L} * \boldsymbol{W} * \boldsymbol{H}$ & Rectangular Solid & $\begin{array}{c}3 \text { Dimensions are } \\
\text { proportional wrt } \\
\text { tumor growth }\end{array}$ \\
\hline $\boldsymbol{L} * \boldsymbol{W}^{2}$ & Rectangular Solid & $\mathrm{H}=\mathrm{W}$ \\
\hline$\frac{\mathbf{1}}{\mathbf{2}} * \boldsymbol{L} * \boldsymbol{W} * \boldsymbol{H}$ & Ellipsoid & $\pi=3$ \\
\hline$\frac{1}{2} * L * W^{2}$ & Ellipsoid & $\mathrm{H}=\mathrm{W}$ \\
\hline $\boldsymbol{L} * \boldsymbol{W}$ & Areal & $\begin{array}{l}\text { Area proportional } \\
\text { to volume }\end{array}$ \\
\hline$\frac{\pi}{4} * L * W$ & Areal & $\begin{array}{l}\text { Area proportional } \\
\text { to volume }\end{array}$ \\
\hline $\boldsymbol{L}$ & Diameter & $\begin{array}{c}\text { Diameter to be } \\
\text { representative to } \\
\text { volume }\end{array}$ \\
\hline$\frac{L+W}{2}$ & Diameter & $\begin{array}{c}\text { Diameter to be } \\
\text { representative to } \\
\text { volume }\end{array}$ \\
\hline
\end{tabular}

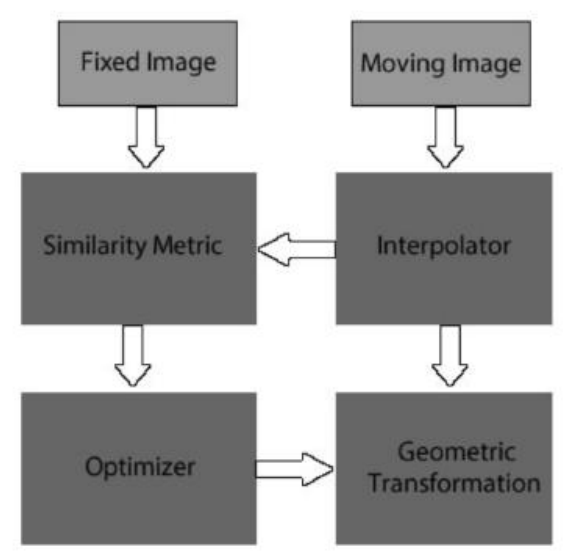

Fig.2. Visual representation of image registration 


\subsection{Geometrical Transformation}

Image registration process has a variety of characteristics. Transformation type is one of the basic characteristic of the image registration in order to properly overlay fixed and moving images. In this section of the paper procedure of selecting the transformation type for our specific application is explained. Affine transformation is an efficient transformation type for this problem now that an affine transformation is composed of a combination of a translation, a rotation, a scale and a shear change. Possible misalignment for MR images taken at different type with the same sensors are translation, rotation scale and shear change.

It is convenient to start by considering linear functions $x, y$ and transformations defined by $x$ and $y$ functions. These transformations might be applied to a point $P(x, y)$ within a plane. All linear transformations $T$ might be represented using following equations:

$$
\begin{aligned}
& x^{\prime}=a x+b y+e \\
& y^{\prime}=c x+d y+f
\end{aligned}
$$

The point $Q\left(x^{\prime}, y^{\prime}\right)$ is called image of $P$ under the transformation $T$. It is written as, $Q=T(P)$. Two equations can be written in matrix form as follow:

$$
\left[\begin{array}{l}
x^{\prime} \\
y^{\prime}
\end{array}\right]=\left[\begin{array}{ll}
a & b \\
c & d
\end{array}\right]\left[\begin{array}{l}
x \\
y
\end{array}\right]+\left[\begin{array}{l}
e \\
f
\end{array}\right]
$$

Two equations can also be written as $Q=M P+\overrightarrow{\mathrm{v}}$, where $M$ and $\vec{v}$ are:

$$
M=\left[\begin{array}{ll}
a & b \\
c & d
\end{array}\right], \quad \vec{v}=\left[\begin{array}{l}
e \\
f
\end{array}\right]
$$

Therefore the product of the matrix $M$ and point $P$ yields $M P$, and the addition of vector $\vec{v}$ and product $M P$ results in a point that is geometrically the transportation of the point by the magnitude and orientation of the vector.

\subsection{Similarity Measure}

The purpose of using the similarity metric is to measure how similar the two images look to each other. Sum of squared differences (SSD) similarity metric is used as the similarity metric in this study. This metric is commonly used in monomodal intensity based image processing tasks. If the registered images are similar to each other, SSD is a proper similarity metric. In monomodal intensity based image processing processes the registered images are generally similar to each other.

\subsubsection{Sum of Squared Differences (SSD)}

If $A$ is the Fixed Image and B Moving Image, then the SSD is expressed as follows:

$$
S S D=\frac{1}{N} \sum_{i}^{N}\left|A(I)-B^{\prime}(I)\right|^{2}, \forall i \in A \cap B^{\prime}
$$

In this equation $A(i)$ is Fixed Image pixel intensity value, $B^{\prime}(i)$ is Moving Image pixel intensity value and $N$ is number of pixels of the images.

It was assumed that the fixed image and the moving image are partially similar to each other. They differ from each other's just because of some misalignments and the target is to minimize those misalignments. In theory, when those misalignments are completely corrected, the measure of similarity value (SSD) becomes zero. SSD is used in this study now that brain MR image registered belongs to the same patients which means the registered images are similar to each other except of the misalignments.

\subsection{Optimizer}

The function of optimizer is to minimize the value of the similarity metric. Therefore, the optimization process ends when the value of the similarity measure is minimum. Consequently, the registration process can be expressed mathematically as follows:

$\min _{T} D[A(i), T(B(i))]$

where

$D=$ Similarity Metric (Cost Function)

$A(i)=$ Fixed Image

$B(i)=$ Moving Image

$T=$ Transformation

\subsubsection{Regular Step Gradient Descend Optimizer}

Regular Step Gradient Method which was found by Cauchy (1847) is used as the optimization type at this study. This method is very commonly used in medical image registration problems thanks to its simplicity. Cauchy was the first to make use of the negative gradient direction in 1847 for minimization problems. In this method an initial trial point $X_{1}$ is chosen, which is iteratively moved along the steepest descent direction until the minimum point is found. Theoretically this method will not terminate unless a stationary point is found. We calculate the difference function at all points in a small (say, $3 \times 3$ ) neighborhood of $X_{k}$ and takes as the next guess $X_{k+1}$ that point which minimizes the difference function.

\subsubsection{Color Based Image Segmentation of Grown,} Diminishing and Unchanged Tumor Parts using $L * a * b *$ Color Space

In CIE $\mathrm{L}^{*} \mathrm{a} \mathrm{b}^{*}$ color space, the vertical axis $L^{*}$ stands for "Lightness or Luminosity"' and its range is $0-100$. The first horizontal axis which is represented by $a^{*}$ stands for colors fall along the red-green axis. The idea is that a color cannot be both red and green [47]. In practice its range is from -128 to +127 (256 levels). The $a^{*}$ axis is red at one end (indicated by $+\mathrm{a}$ ), and green at the other end (indicated by $-\mathrm{a}$ ). The other horizontal axis which is represented by $b^{*}$ stands for colors fall along the blue-yellow axis. The idea is again that a color cannot be both blue and yellow. In practice its range is from -128 to +127 (256 
levels). The $b^{*}$ axis is yellow at one end (indicated by $+\mathrm{b}$ ), and blue at the other end (indicated by -b). The origin of each axis is 0 . A value of 0 or very low numbers of both $a^{*}$ and $b^{*}$ will define a neutral or near neutral. $a^{*}$ and $b^{*}$ layers contain color information whereas $L^{*}$ layer contains luminosity (lightness) information [48].

Considering all the properties and advantages of CIE $L * a * b^{*}$ up to now, it can be concluded that the difference between the two points in the CIE $L^{*} a^{*} b^{*}$ color space is same with the human visual system. Therefore, after image registration process, obtained medical images are converted to $C I E L^{*} a^{*} b^{*}$ from $R G B$ color space. Conversion from $X Y Z$ color space to CIE $L^{*} a^{*} b^{*}$ color space is achieved using the equation 8-9.

$$
\left(\begin{array}{c}
L^{*}=116 f\left(\frac{Y}{Y_{n}}\right)-16 \\
a^{*}=500\left[f\left(\frac{X}{X_{n}}\right)-f\left(\frac{Y}{Y_{n}}\right)\right] \\
b^{*}=200\left[f\left(\frac{Y}{Y_{n}}\right)-f\left(\frac{Z}{Z_{n}}\right)\right]
\end{array}\right)
$$

where,

$$
f(t)=\left\{\begin{array}{cc}
t^{1 / 3}, & t>\left(\frac{6}{29}\right)^{3} \\
\frac{1}{3}\left(\frac{29}{6}\right)^{2} t+\frac{4}{29}, & \text { otherwise }
\end{array}\right.
$$

$X, Y$ and $Z$ are the coordinates of $X Y Z$ color space. $X_{n}, Y_{n}$, and $Z_{n}$ are $X Y Z$ tristimulus values of the reference white point. The subscript $n$ stands for "normalized". The reason for partition off $f(t)$ function is to prevent an infinite slope at $t=0$.

\subsubsection{Color Differences, Delta E Differences and Tolerances}

In this study color difference is used to segment grown, diminishing and unchanged tumor parts from rest of the image and from each other as well after images are registered. Color difference is a well-advised technique to compute difference (distance) between two colors in color based image segmentation science. Color difference is a kind of metric which actually provides Euclidean distance. Delta E was defined by The International Commission on Illumination (CIE) and represented by $\Delta E$ which generally indicates color difference. The higher the $\Delta E$, the bigger the difference between two colors in comparison. Theoretically, for average human vision a $\Delta E$ less than 1 is said to be indistinguishable on the condition that colors are not adjacent to each other. This means that color difference of less than 1 is hardly distinguishable by average human vision. a $\Delta E$ value between 3 and 6 is supposed to be moderate [47]. $\Delta E$ is computed using equation 10 .

$$
\Delta E=\sqrt{\left(L_{2}^{*}-L_{1}^{*}\right)^{2}+\left(a_{2}^{*}-a_{1}^{*}\right)^{2}+\left(b_{2}^{*}-b_{1}^{*}\right)^{2}}
$$

$\left(L_{1}^{*}, a_{1}^{*}, b_{1}^{*}\right)$ and $\left(L_{2}^{*}, a_{2}^{*}, b_{2}^{*}\right)$ are two points having three components: $L^{*}, a^{*}, b^{*}$ in three dimensional CIE $L^{*} a^{*} b^{*}$ color space.

Tolerance means that how a set of colors is close to a specified reference point. Now that the distance in $L^{*} a^{*} b^{*}$ color space is perceptually uniform, tolerance will be defined as the set of colors whose difference to the reference point is smaller than noticeable-difference threshold. This tolerance value will specify the cluster of similar colors, i.e. pixel values. Tolerance value is a quality control for segmenting colors from each other, hence shows difference (distance) for color and lightness.

The whole process including image registration and image segmentation is summarized as follows:

\section{Proposed Algorithm:}

Step 1: Read the patient's MR image with brain tumor taken at a previous time and save as Fixed Image. Read the patient's MR image with brain tumor taken at a different time and save as Moving Image.

Step 2: Register Fixed and Moving Images using similarity metric and optimizer defined previously.

Step 3: Save Fused (registered) image.

Repeat Steps 1-3 for all tumor associated MR scans of the patient brain.

Step 4: Convert Fused medical images from $R G B$ color space to $C I E L^{*} a^{*} b^{*}$ color space using equations 8-9. In $C I E$ $L^{*} a^{*} b^{*}$ color space, the vertical axis $L^{*}$ stands for "Lightness or Luminosity". The first horizontal axis which is represented by $a^{*}$ stands for colors fall along the red-green axis. The other horizontal axis which is represented by $b^{*}$ stands for colors fall along the blue-yellow axis.

Step 5: Draw free-hand irregularly shaped region to specify a color (i.e. anatomic parts: grown tumor, diminishing tumor or unchanged tumor).

Step 6: Compute Color Difference (Delta E) for every pixel in the image between that pixel's color and the average $C I E$ $L^{*} a * b^{*}$ color of the drawn region using equation 10.

Step 7: Specify the Tolerance Value according to sensitivity your work needs. Tolerance value is a quality control for segmenting colors from each other, hence shows difference (distance) for color and lightness. This is a number that indicates how close to that color would the user like to be. The algorithm then will find all pixels within that computed Delta E of the color of the drawn region.

Step 8: Categorize each pixel using nearest neighbor idea which tells that the smallest distance means similar colors, hence similar anatomic parts.

Step 9: Create new image that segment the original image by color. Green color shows tumor which has been growing with time. Magenta color, on the other hand, shows tumor which has been diminishing parts with time and lastly white color shows unchanged brain tumors.

Step 10: Compute the area of each color (each anatomic part) in segmented image.

Repeat Steps 4-10 for all Fused (registered) images. 
Step 11: Add all the results came from Step 10 to compute volume of grown brain tumor, diminished brain tumor and unchanged brain tumor.

\section{EXPERIMENTAL RESULTS}

The dataset that is used in this study is a publicly available dataset [49] and is known as RIDER Neuro MRI project from The Cancer Imaging Archive (TCIA) database [50]. This is a cancer research project which aims to collect as much as possible brain MR images of the patients with tumors in the brain. This dataset includes 70,220 MR images taken from 19 patients with recurrent glioblastoma.

Figure 3(left) (Fixed Image) and right (Moving Image) are MR images of a patient brain that has brain tumor. Tumors are marked with red arrows in the associated images. These MR images are taken at two different times. Figure 3(left) and (right) are just one scan of the patient acquired at different times. However, registration process has been applied to all scans which have brain tumor. In this patient 30 scans of the patient brain have brain tumor. Slices thickness between scans is $1 \mathrm{~mm}$ which is a perfect thickness for tumor analysis. It has been investigated experimentally how the brain tumor grows, specifically which part of the brain tumor grows, diminishes, or un-changes with time.
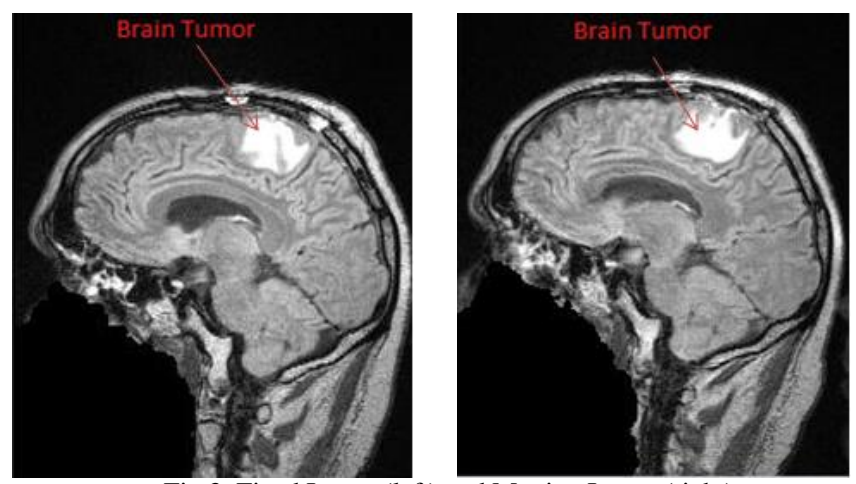

Fig.3. Fixed Image (left) and Moving Image (right)
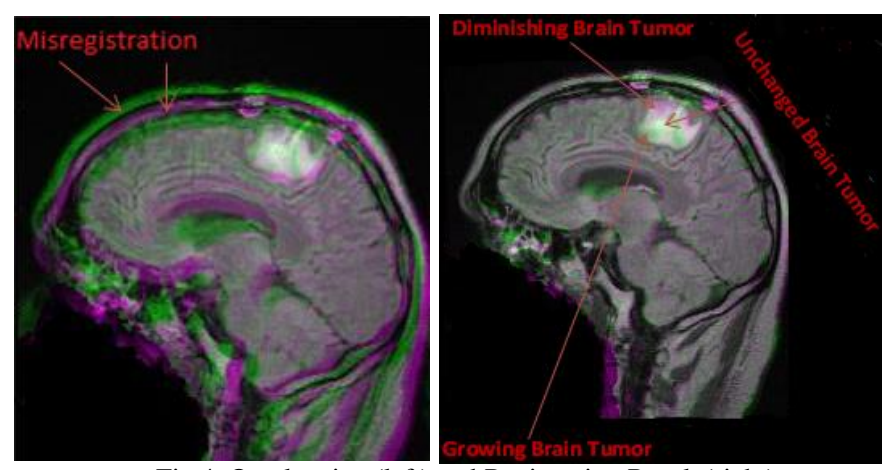

Fig.4. Overlapping (left) and Registration Result (right)

Figure 4(left) is just overlapping of two scans. Misregistration of the scans is quite obvious. Misregistration between two scans is marked with red arrows as well. Figure 4(right) is registration result. In this figure, it can be seen that distortions which is called misregistration is removed. The remaining variations are changes which are of interest; they are therefore not distortions; they are tumor changes which are desired to be detected. These important changes are marked with red arrows. Green parts show tumor which has been growing with time. Magenta parts, on the other hand, shows tumor which has been diminishing parts with time and lastly white parts are unchanged brain tumors. This process has been applied to all 30 scans and results can be seen in Figure 5 and Figure 6.

Segmented tumor after registration process is individually indicated in Figure 5(left). Figure 5(right) is filtering result of segmented tumor image. Figure 5(right) is necessary to compute area (hence volume) of diminished tumor part, growing tumor part and unchanged tumor part on an individual basis.

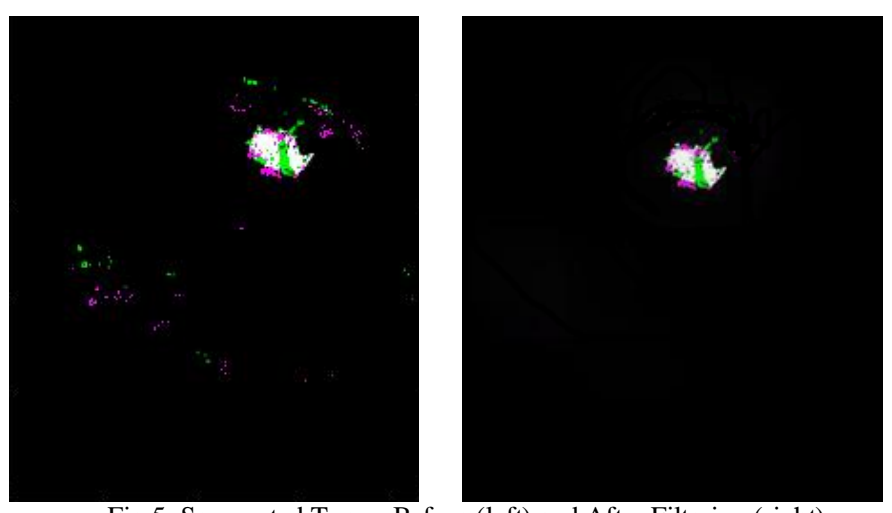

Fig.5. Segmented Tumor Before (left) and After Filtering (right)

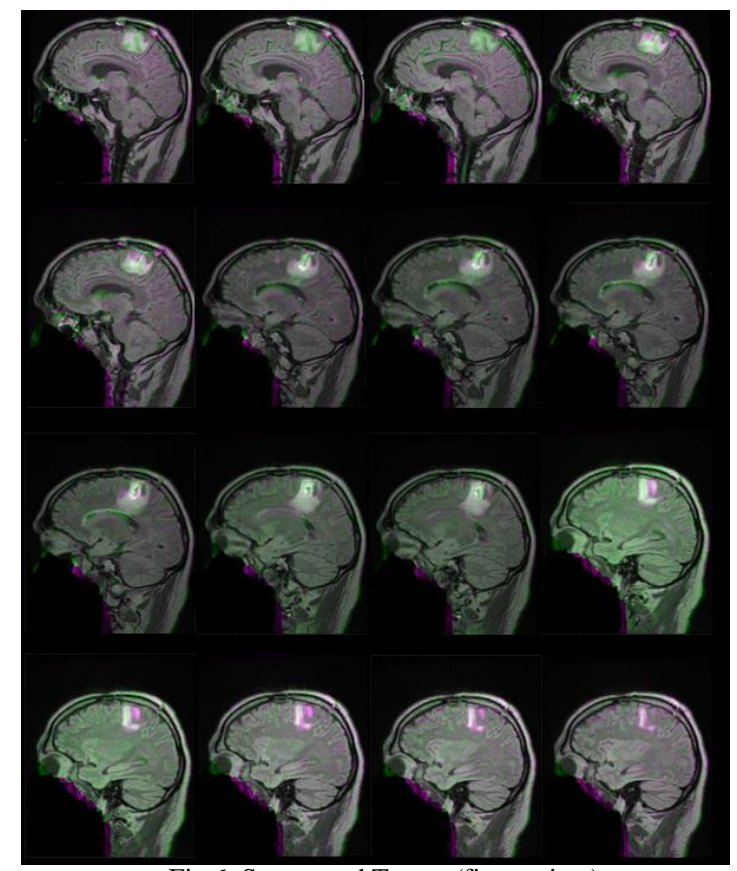

Fig.6. Segmented Tumor (first patient)

Scores related to SSD metric is tabulated in Table II. When the iteration number increases the better match is achieved. Our rule is that the registration is better when the SSD is lower. Looking at the Table II it is seen that the best match is found at the Iteration Number 86. At 86th iteration number the value of SSD is 286.2024 whereas SSD number starts with 1627.2952 at 1 st iteration number. However, the time required for the 
registration process increases when the number of iterations increase. Hence registration process takes longer time when iteration number increases.

As stated before the process explained until this point has been implemented to all tumor associated part of the brain. For first patient this number was 30 scans. For demonstration, result of 16 scans is shown in Figure 6 and corresponding tumors are shown in Figure 7.

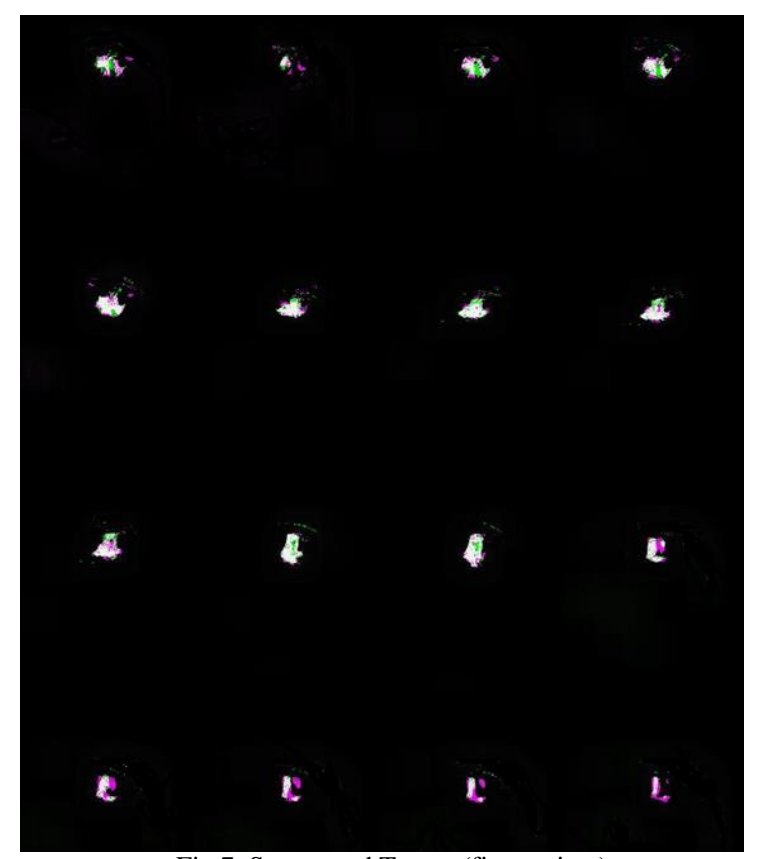

Fig.7. Segmented Tumor (first patient)

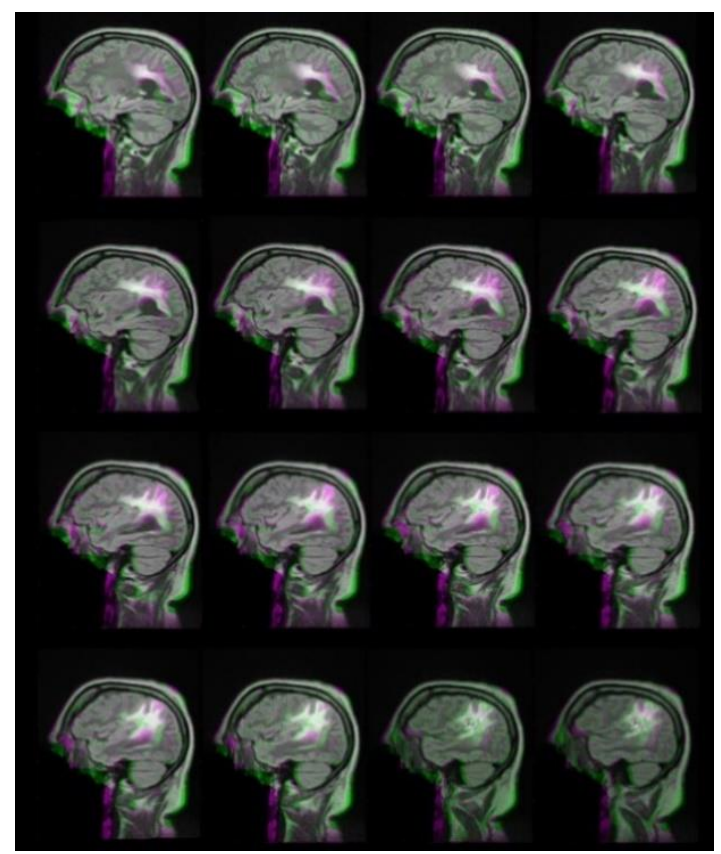

Fig.8. Segmented Tumor (second patient)

Results for the second patient are shown in Figure 8 and Figure 9. For second patient, number of scans which are tumor associated part of the brain was 24. Registration process has been applied to 24 scans. For demonstration, result of 16 scans is shown in Figure 8 and corresponding tumors are shown in Figure 9.
TABLE II

SSD RESULTS WITH RESPECT TO ITERATION NUMBER

\begin{tabular}{|c|c|c|c|c|c|}
\hline Iter. SSD & Iter. SSD & Iter. & SSD & Iter. & SSD \\
\hline $1 \quad 1627.2952$ & $26 \quad 321.2599$ & 51 & 286.8984 & 76 & 287.2046 \\
\hline $2 \quad 1268.3555$ & $27 \quad 317.4207$ & 52 & 286.8578 & 77 & 286.6178 \\
\hline $3 \quad 1584.4344$ & $28 \quad 314.0301$ & 53 & 286.6623 & 78 & 286.5840 \\
\hline $4 \quad 5242.0876$ & 29310.8376 & 54 & 286.6876 & 79 & 286.5539 \\
\hline $5 \quad 2098.3634$ & $30 \quad 307.9623$ & 55 & 286.7207 & 80 & 286.6160 \\
\hline $6 \quad 1851.5011$ & $31 \quad 305.6251$ & 56 & 286.6427 & 81 & 286.5475 \\
\hline $7 \quad 1525.9047$ & $32 \quad 303.2605$ & 57 & 286.5345 & 82 & 286.7101 \\
\hline $8 \quad 1354.2674$ & $33 \quad 300.5287$ & 58 & 286.5914 & 83 & 286.6453 \\
\hline 834.4523 & $34 \quad 298.3436$ & 59 & 286.3135 & 84 & 286.7862 \\
\hline $10 \quad 665.7449$ & $35 \quad 296.8114$ & 60 & 286.6338 & 85 & 286.8951 \\
\hline $11 \quad 617.4345$ & $\begin{array}{ll}36 & 294.9558 \\
\end{array}$ & 61 & 286.2620 & 86 & 286.2024 \\
\hline $12 \quad 584.5832$ & $37 \quad 293.3388$ & 62 & 286.3391 & 87 & CONVERGE \\
\hline $13 \quad 550.9578$ & $38 \quad 292.7274$ & 63 & 286.7855 & 88 & CONVERGE \\
\hline $14 \quad 520.9090$ & $39 \quad 290.8807$ & 64 & 287.3632 & 89 & CONVERGE \\
\hline $15 \quad 489.3432$ & $40 \quad 291.0479$ & 65 & 290.5421 & 90 & CONVERGE \\
\hline $16 \quad 461.6606$ & $41 \quad 294.3186$ & 66 & 286.9279 & 91 & CONVERGE \\
\hline $17 \quad 432.3264$ & $42 \quad 301.4828$ & 67 & 286.4376 & 92 & CONVERGE \\
\hline $18 \quad 409.9917$ & $43 \quad 302.8219$ & 68 & 286.2334 & 93 & CONVERGE \\
\hline $19 \quad 386.5456$ & $44 \quad 290.8027$ & 69 & 286.2593 & 94 & CONVERGE \\
\hline $20 \quad 386.8915$ & $45 \quad 287.8876$ & 70 & 286.3713 & 95 & CONVERGE \\
\hline $21 \quad 484.6777$ & $46 \quad 287.7257$ & 71 & 286.3901 & 96 & CONVERGE \\
\hline $22 \quad 579.0272$ & $47 \quad 287.5535$ & 72 & 286.4022 & 97 & CONVERGE \\
\hline $23 \quad 433.4253$ & $48 \quad 287.3056$ & 73 & 286.4613 & 98 & CONVERGE \\
\hline $24 \quad 338.2038$ & $49 \quad 287.1376$ & 74 & 286.8364 & 99 & CONVERGE \\
\hline $25 \quad 325.1858$ & $50 \quad 287.1037$ & 75 & 286.6227 & 100 & CONVERGE \\
\hline
\end{tabular}

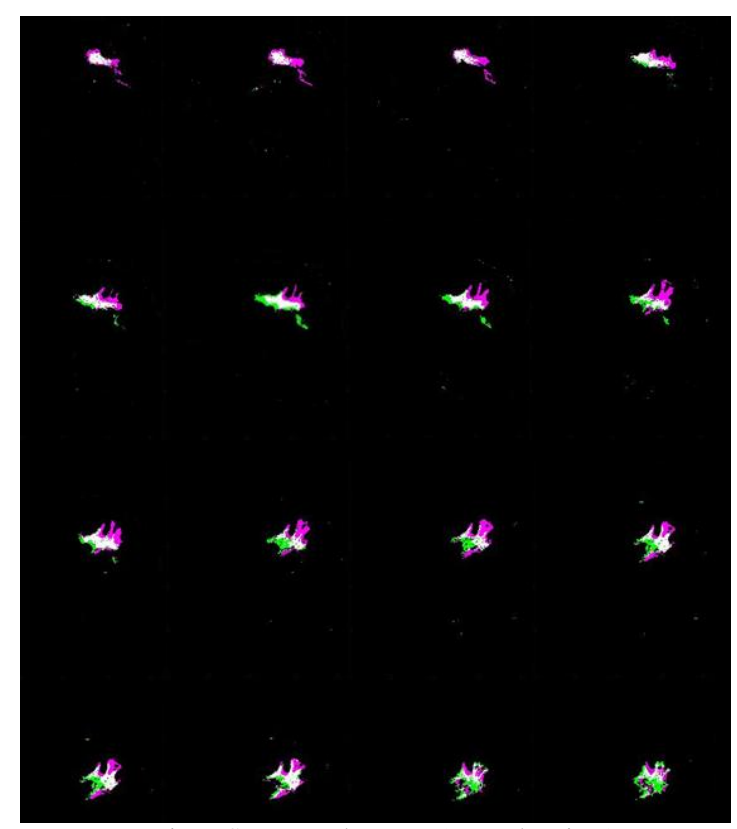

Fig.9. Segmented Tumor (second patient)

Table III shows volume measurement results for both mathematical models and medical image registration model. Table III is comparison of the proposed method with other 
standard methods. For tumor volume evaluation, there exist important mathematical models. Various studies show that three dimensional fundamental shape of brain tumor is hemiellipsoid. Table III shows results for 12 mathematical formula and medical image registration-segmentation method. Through these mathematical formulas $\pi / 6^{*} L^{*} W^{*} H$ is the most used formula for tumor size variation volume measurement. Results of mathematical formulas can vary from formula to formula. Results of tumor volume vary between $72654 \mathrm{~mm}^{3}$ and 16474 $\mathrm{mm}^{3}$. Through these mathematical formulas $\pi / 6^{*} L^{*} W^{*} H$ is the most used formula for tumor size variation volume measurement and with this formula result is found to be 36659 $\mathrm{mm}^{3}$. Medical image registration-segmentation result is found to be $36154 \mathrm{~mm}^{3}$. This proves that medical image-registration method is not only between the true ranges but also is very close to the best formula. In reality medical image registrationsegmentation formula gives better result than all mathematical formulas including $\pi / 6^{*} L^{*} W^{*} H$ formula. The reason is that all mathematical formulas make some assumption when measuring tumor volume. However medical image registrationsegmentation method does not make any assumption. It computes each MR scan with $1 \mathrm{~mm}$ thickness and adds all results to compute volume. This gives the most real volumes for tumor. Besides, growing tumor part, diminishing tumor part and unchanged tumor part are also possible just for medical image registration-segmentation method.

TABLE III

TUMOR VOLUME MEASUREMENT RESULT

\begin{tabular}{|c|c|c|c|c|c|c|}
\hline Formula Used & $\begin{array}{c}\text { Previous } \\
\text { Tumor } \\
\text { Volume } \\
\left(\mathrm{mm}^{3}\right) \\
\end{array}$ & $\begin{array}{c}\text { Current } \\
\text { Tumor } \\
\text { Volume } \\
\left(\mathrm{mm}^{3}\right) \\
\end{array}$ & $\begin{array}{c}\text { Growing } \\
\text { Tumor } \\
\text { Volume } \\
\left(\mathrm{mm}^{3}\right) \\
\end{array}$ & $\begin{array}{c}\text { Diminishing } \\
\text { Tumor } \\
\text { Volume } \\
\left(\mathrm{mm}^{3}\right)\end{array}$ & $\begin{array}{c}\text { Unchanged } \\
\text { Tumor } \\
\text { Volume } \\
\left(\mathrm{mm}^{3}\right)\end{array}$ & $\begin{array}{l}\text { Difference } \\
\quad\left(\mathbf{m m}^{3}\right)\end{array}$ \\
\hline $\begin{array}{l}\text { Medical Image Registration- } \\
\text { Segmentation Method }\end{array}$ & 36154 & 31221 & 10345 & 15278 & 20876 & (-) 4933 \\
\hline$\frac{\pi}{6} * L * W * H$ & 36659 & 31653 & N/A & N/A & N/A & (-) 5006 \\
\hline$\frac{\pi}{6} * L * W^{2}$ & 34709 & 27689 & N/A & N/A & N/A & (-) 7020 \\
\hline$\frac{\pi}{6} *\left[\frac{L * W}{2}\right]^{3}$ & 46678 & 41601 & N/A & N/A & N/A & (-) 5077 \\
\hline$\frac{\pi}{6} *(L * W)^{\frac{3}{2}}$ & 46689 & 41673 & N/A & N/A & N/A & (-) 5016 \\
\hline $0.4^{*} \boldsymbol{L} * \boldsymbol{W}^{2}$ & 28033 & 21177 & N/A & N/A & N/A & (-) 6856 \\
\hline$\frac{\mathbf{4}}{\mathbf{3}} * \pi *\left(\frac{L+\boldsymbol{W}}{2}\right)^{3}$ & 34543 & 29762 & N/A & N/A & N/A & $(-) 4781$ \\
\hline$\frac{\mathbf{4}}{\mathbf{3}} * \boldsymbol{\pi} *\left(\frac{L}{\mathbf{2}}\right)^{3}$ & 72654 & 45652 & N/A & N/A & N/A & (-) 27012 \\
\hline $\boldsymbol{L} * \boldsymbol{W} * \boldsymbol{H}$ & 70049 & 60483 & N/A & N/A & N/A & (-) 9566 \\
\hline $\boldsymbol{L} * \boldsymbol{W}^{2}$ & 66322 & 52909 & N/A & N/A & N/A & (-) 13423 \\
\hline$\frac{1}{2} * L * W * H$ & 35024 & 30241 & N/A & N/A & N/A & (-) 4783 \\
\hline$\frac{\mathbf{1}}{\mathbf{2}} * \boldsymbol{L} * \boldsymbol{W}^{2}$ & 33166 & 26454 & N/A & N/A & N/A & (-) 6712 \\
\hline $\boldsymbol{L} * \boldsymbol{W}$ & 20987 & 15654 & N/A & N/A & N/A & (-) 5333 \\
\hline$\frac{\pi}{4} * L * W$ & 16474 & 12288 & N/A & N/A & N/A & (-) 4186 \\
\hline
\end{tabular}


TABLE IV

TUMOR SIZE VARIATION RESULTS FOR 19 PATIENTS USING MEDICAL IMAGE REGISTRATION-SEGMENTATION METHOD

\begin{tabular}{|c|c|c|c|c|c|c|}
\hline $\begin{array}{l}\text { Patient } \\
\text { Number }\end{array}$ & $\begin{array}{l}\text { Previous } \\
\text { Tumor } \\
\text { Volume } \\
\quad\left(\mathbf{m m}^{3}\right)\end{array}$ & $\begin{array}{l}\text { Current } \\
\text { Tumor } \\
\text { Volume } \\
\quad\left(\mathrm{mm}^{3}\right)\end{array}$ & $\begin{array}{l}\text { Growing } \\
\text { Tumor } \\
\text { Volume } \\
\quad\left(\mathrm{mm}^{3}\right)\end{array}$ & $\begin{array}{l}\text { Diminishing } \\
\text { Tumor } \\
\text { Volume } \\
\quad\left(\mathrm{mm}^{3}\right)\end{array}$ & $\begin{array}{l}\text { Unchanged } \\
\text { Tumor } \\
\text { Volume } \\
\quad\left(\mathrm{mm}^{3}\right)\end{array}$ & $\begin{array}{l}\text { Difference } \\
\left(\mathbf{m m}^{3}\right)\end{array}$ \\
\hline 1. Patient & 36154 & 31221 & 10345 & 15278 & 20876 & (-) 4933 \\
\hline 2. Patient & 24356 & 21967 & 3924 & 6313 & 18043 & (-) 2389 \\
\hline 3. Patient & 37234 & 38644 & 15432 & 14022 & 23212 & (+) 1410 \\
\hline 4. Patient & 18465 & 20087 & 2223 & 601 & 17864 & (+) 1622 \\
\hline 5. Patient & 23987 & 29647 & 10002 & 4342 & 19645 & (+) 5660 \\
\hline 6. Patient & 34123 & 31076 & 9109 & 12156 & 21967 & (-) 3047 \\
\hline 7. Patient & 26781 & 25816 & 9081 & 10046 & 16735 & (-) 965 \\
\hline 8. Patient & 21647 & 19087 & 184 & 2744 & 18903 & (-) 2560 \\
\hline 9. Patient & 42790 & 44718 & 14873 & 12945 & 29845 & (+) 1928 \\
\hline 10. Patient & 20043 & 25098 & 10055 & 5000 & 15043 & (+) 5055 \\
\hline 11. Patient & 34981 & 30241 & 7903 & 12643 & 22338 & (-) 4740 \\
\hline 12. Patient & 22132 & 27457 & 10048 & 4723 & 17409 & (+) 5325 \\
\hline 13. Patient & 30483 & 35654 & 10531 & 5360 & 25123 & (+) 5171 \\
\hline 14. Patient & 38654 & 33376 & 11411 & 16689 & 21965 & (-) 5278 \\
\hline 15. Patient & 27908 & 31209 & 10319 & 7018 & 20890 & (+) 3301 \\
\hline 16. Patient & 17592 & 20982 & 4237 & 847 & 16745 & (+) 3390 \\
\hline 17. Patient & 23879 & 18231 & 2485 & 8133 & 15746 & (-) 5648 \\
\hline 18. Patient & 30675 & 26783 & 11110 & 15002 & 15673 & (-) 3892 \\
\hline 19. Patient & 29876 & 33832 & 13748 & 9792 & 20084 & (+) 3956 \\
\hline
\end{tabular}

Results for 19 patients' brain tumor size variation volumes using medical image registration-segmentation method are demonstrated in Table IV.

\section{CONCLUSION}

A useful and effective application of medical image registration-segmentation is offered in this paper with comparison of mathematical based methods. Intensity-based medical image registration phenomenon is used in this study. Sum of squared differences metric is used as similarity metric and regular step gradient descent optimizer is used as optimization technique. $L * a * b$ color space image segmentation is used to segment each part of tumor. Tumor growthiness inside the patient's brain is successfully investigated. For the first patient, results are shown in Figure 6 and Figure 7. Grown brain tumor volume is found to be $10345 \mathrm{~mm}^{3}$, diminished brain tumor volume is found to be $15278 \mathrm{~mm}^{3}$ and unchanged brain tumor volume is found to be $20876 \mathrm{~mm}^{3}$. Process is applied to another patient and results are shown in Figure 8, Figure 9. For the second patient, grown brain tumor volume is found to be $11657 \mathrm{~mm}^{3}$, diminished brain tumor volume is found to be $14657 \mathrm{~mm}^{3}$ and unchanged brain tumor volume is found to be $18076 \mathrm{~mm}^{3}$. Technique is implemented to 19 patients and satisfactory results are obtained and demonstrated in Table IV. A very useful aspect of medical image registrationsegmentation method for brain tumor investigation is that grown, diminished, and unchanged brain tumor parts of the patients are investigated and computed on an individual basis in a three-dimensional manner within the time. On the other hand, there is no possibility of mathematical based methods to computer grown, diminished and unchanged tumor parts. Mathematical based methods can compute previous tumor volume and next tumor volume. Most mathematical based methods are reasonable for tumor volume measurement but medical image registration is more accurate because it measures actual volume without making any assumptions.

Besides the advantages of the proposed method it also has some restriction. For instance, the input size of the MR images in the dataset must be the same for the registration algorithm to work successfully. In addition, it is still a challenge for the proposed method to deal with low-resolution images. In the future work in this area, the pre-processing image enhancement method may be added to the proposed algorithm. Tumor volume measurement for other organs of the body may be another future work using the proposed method in this paper.

\section{REFERENCES}

[1] L.M. DeAngelis, "Brain tumors", Med. Prog. N Engl J Med., 114(1), 2001, 114-123.

[2] B. A. Kohler et al., "Annual report to the nation on the status of cancer, 1975-2007, featuring tumors of the brain and other nervous system", J. Natl. Cancer Inst. 103(9), 2011, 714-736.

[3] G. Mazzara, R. Velthuizen, J. Pearlman, H. Greenberg, H. Wagner, "Brain tumor target volume determination for radiation treatment planning through automated MRI segmentation", Int J Radiat Oncol Biol Phys. 59(1), 2004, 300-312.

[4] F.K. Landeghem et al., "Post-mortem studies in glioblastoma patients treated with thermotherapy using magnetic nanoparticles", Biomaterials. 30(1), 52-57.

[5] D. Krex et al., "Long-term survival with glioblastoma multiforme" Brain, 130(10), 2007, 2596-2606. 
[6] R.B. Seither, B. Jose, K.J. Paris, R.D. Lindberg, W.J. Spanos, "Results of irradiation in patients with high-grade gliomas evaluated by magnetic resonance imaging", Am. J. Clin. Oncol., 18(4), 1995, 297-299.

[7] J.M. Caudrelier et al., "MRI definition of target volumes using fuzzy logic method for three-dimensional conformal radiation therapy", Int. J. Radiat. Oncol. Biol. Phys. 55(1), 2003, 225-233.

[8] R.K. T. Haken et al., "A quantitative assessment of the addition of MRI to CT-based, 3-D treatment planning of brain tumors", Radiother. Oncol. 25(2), 1992, 121-133.

[9] E.C. Halperin, G. Bentel, E.R. Heinz, P.C. Burger, "Radiation therapy treatment planning in supratentorial glioblastoma multiforme: an analysis based on post mortem topographic anatomy with CT correlations", Int. J. Radiat. Oncol. Biol. Phys., 17(6), 1989, 1347-1350.

[10] K. Kaplan, Y. Kaya, M. Kuncan, H. M. Ertunc, "Brain tumor classification using modified local binary patterns (LBP) feature extraction methods", Medical Hypotheses, 139 (2020), 109696.

[11] V.S. Khoo, E.J. Adams, F. Saran, J.L. Bedford, J.R. Perks, A.P. Warrington, M. Brada, "A comparison of clinical target volumes determined by CT and MRI for the radiotherapy planning of base of skull meningiomas", Int. J. Radiat. Oncol. Biol. Phys., 46(5), 2000, 1309-1317.

[12] P. Sminia, R. Mayer, "External beam radiotherapy of recurrent glioma: radiation tolerance of the human brain", Cancers (Basel). 4(2), 2012, 379 399.

[13] R.K. Ten Haken, B.A. Fraass, A.S. Lichter, L.H. Marsh, E.H. Radany, H.M. Sandler, "A brain tumor dose escalation protocol based on effective dose equivalence to prior experience", Int. J. Radiat. Oncol. Biol. Phys. 42(1), 1998, 137-141.

[14] D.H. Char, S. Kroll, T.L. Phillips, "Uveal melanoma: growth rate and prognosis", Arch. Ophthalmol. 115(8), 1997, 1014-1018.

[15] J.M. Romero, P.T. Finger, R.B. Rosen, R. Iezzi, "Three-dimensional ultrasound for the measurement of choroidal melanomas", Arch. Ophthalmol. 119(9), 2001, 1275-1282.

[16] T. Grasbon, S. Schriever, J.P. Hoops, A.J. Mueller, "3D-Ultraschall Erste Erfahrungen bei verschiedenen Augenerkrankungen", Der Ophthalmol. 98(8), 2001, 88-93.

[17] W. Li, E.S. Gragoudas, K.M. Egan, "Tumor basal area and metastatic death after proton beam irradiation for choroidal melanoma", Arch. Ophthalmol. 121(1), 2003, 68-72.

[18] E. Richtig, G. Langmann, K. Müllner, G. Richtig, J. Smolle, “Calculated tumour volume as a prognostic parameter for survival in choroidal melanomas", Eye (Lond). 18(6), 2004, 619-623.

[19] Y. Liu, S.M. Sadowski, A.B. Weisbrod, E. Kebebew, R.M. Summers, J. Yao, "Patient specific tumor growth prediction using multimodal images", Med. Image Anal. 18(3), 2014, 555-566.

[20] R. Rask, P.K. Jensen, "Precision of ultrasonic estimates of choroidal melanoma regression" 233(1995), 1995, 777-782.

[21] H. Rubin, P. Arnstein, B.M. Chu, "Tumor progression in nude mice and its representation in cell culture", J. Natl. Cancer Inst. 77(5), 1986, 11251135.

[22] H. Rubin, B.M. Chu, P. Arnstein, "Selection and adaptation for rapid growth in culture of cells from delayed sarcomas in nude mice", Cancer Res. 47(2), 1987, 486-492.

[23] S. Karpagam, S. Gowri, "Brain tumor growth and volume detection by ellipsoid-diameter technique using MRI data", Int. J. Comput. Sci. 9(2012), 2012, 121-126.

[24] M.F. Dempsey, B.R. Condon, D.M. Hadley, Measurement of tumor "size" in recurrent malignant glioma: $1 \mathrm{D}, 2 \mathrm{D}$, or 3D?, AJNR Am. J. Neuroradiol. 26 (2005) 770-776.

[25] A. Talkington, R. Durrett, Estimating tumor growth rates in vivo, V (2014) $1-27$.

[26] T. Pala, A.Y. Camurcu, "Design of decision support system in the setastatic colorectal cancer data set and its application", Balkan Journal of Electrical and Computer Engineering 4(1), 2016, 12-16.

[27] H.S. Nogay, T.H. Akinci, "A convolutional neural network application for predicting the locating of squamous cell carcinoma in the lung", Balkan Journal of Electrical and Computer Engineering 6(3), 2018, 207210.

[28] F. Ozyurt, E. Sert, E. Avci and E. Dogantekin, Brain tumor detection based on Convolutional Neural Network with neutrosophic expert maximum fuzzy sure entropy, Measurement, 147, 106830.

[29] S.E.A. Muenzing, B. Ginneken, K. Murphy, J.P.W. Pluim, "Supervised quality assessment of medical image registration: Application to intrapatient CT lung registration", Med. Image Anal. 16(8), 2012, 1521-1531.

[30] K.K. Brock, L.A. Dawson, M.B. Sharpe, D.J. Moseley, D.A. Jaffray, "Feasibility of a novel deformable image registration technique to facilitate classification, targeting, and monitoring of tumor and normal tissue”, Int. J. Radiat. Oncol. Biol. Phys. 64(4), 2006, 1245-1254.

[31] M.R. Kaus, S.K. Warfield, A. Nabavi, P.M. Black, F.A. Jolesz, R. Kikinis, "Automated segmentation of MR images of brain tumors", Radiology, 218(2), 2001, 586-591.

[32] J.P. Thirion, "Image matching as a diffusion process: an analogy with Maxwell's demons", Med. Image Anal. 2(3), 1998, 243-260.

[33] I. Bloch, O. Colliot, O. Camara, T. Géraud, "Fusion of spatial relationships for guiding recognition, example of brain structure recognition in 3D MRI", Pattern Recognit. Lett. 26(4), 2005, 449-457.

[34] B. Alfano, M. Ciampi, G. De Pietro, "A wavelet-based algorithm for multimodal medical image fusion", Int. Conf. Semant. Digit. Media Technol., Springer, 4816(2007), 2007, 117-120.

[35] K. Yuanyuan, L. Bin, T. Lianfang, M. Zongyuan, "Multi-modal medical image fusion based on wavelet transform and texture measure", Control Conf., 2007. Chinese, IEEE, 2007, 697-700.

[36] Q.P. Zhang, M. Liang, W.C. Sun, "Medical diagnostic image fusion based on feature mapping wavelet neural networks", Image Graph. (ICIG'04), Third Int. Conf., IEEE, 2004: pp. 51-54.

[37] Q.P. Zhang, W.J. Tang, L.L. Lai, W.C. Sun, K.P. Wong, "Medical diagnostic image data fusion based on wavelet transformation and selforganising features mapping neural networks, Mach. Learn. Cybern. 2004. Proc. 2004 Int. Conf., IEEE, 2004: pp. 2708-2712.

[38] G. Quellec, M. Lamard, G. Cazuguel, B. Cochener, C. Roux, "Wavelet optimization for content-based image retrieval in medical databases, Med. Image Anal. 14(2), 2010, 227-241.

[39] M. Havaei, A. Davy, D. Warde-Farley, A. Biard, A. Courville, Y. Bengio, C. Pal, P.M. Jodoin, H. Larochelle, "Brain tumor segmentation with deep neural networks", Med. Image Anal. 35(2017), 2017, 18-31.

[40] K.M. Pohl, E. Konukoglu, S. Novellas, N. Ayache, A. Fedorov, I.F. Talos, A. Golby, W.M. Wells, R. Kikinis, P.M. Black, "A new metric for detecting change in slowly evolving brain tumors: Validation in meningioma patients", Neurosurgery. 68(1), 2011, 225-233.

[41] S. Bauer, R. Wiest, L.-P. Nolte, M. Reyes, "A survey of MRI-based medical image analysis for brain tumor studies", Phys. Med. Biol. 58(13), 2013, R97-R129.

[42] E.D. Angelini, J. Delon, A.B. Bah, L. Capelle, E. Mandonnet, "Differential MRI analysis for quantification of low grade glioma growth", Med. Image Anal. 16(1), 2012, 114-126.

[43] K.F. Schmidt, M. Ziu, N.O. Schmidt, P. Vaghasia, T.G. Cargioli, S. Doshi, M.S. Albert, P.M. Black, R.S. Carroll, Y. Sun, "Volume reconstruction techniques improve the correlation between histological and in vivo tumor volume measurements in mouse models of human gliomas", J. Neurooncol. 68(2004), 2004, 207-215.

[44] J.P. Feldman, R. Goldwasser, "A mathematical model for tumor volume evaluation using two-dimensions", Journal Appl. Quant. Methods, 4(4), 2009, 455-462.

[45] M.M. Tomayko, C.P. Reynolds, "Determination of subcutaneous tumor size in athymic (nude) mice", Cancer Chemother. Pharmacol. 24(1989), 1989, 148-154.

[46] X. Du, J. Dang, Y. Wang, S. Wang, T. Lei, "A parallel nonrigid registration algorithm based on B-spline for medical images", Comput. Math. Methods Med. 2016.

[47] P.J. Baldevbhai, R.S. Anand, "Color Image Segmentation for Medical Images using $\mathrm{L} * \mathrm{a} * \mathrm{~b} *$ Color Space", J. Electron. Commun. Eng., 1(2), 2012, 24-45.

[48] V.S. Rathore, M.S. Kumar, A. Verma, "Colour based image segmentation using $\mathrm{L} * \mathrm{~A} * \mathrm{~B} *$ colour space based on genetic algorithm", Int. J. Emerg. Technol. Adv. Eng. 2(6), 2012, 156-162.

[49] D. Barboriak, The cancer imaging archive, doi.org/10.7937/K9/TCIA.2015.VOSN3HN1.

[50] K. Clark, B. Vendt, K. Smith, J. Freymann, J. Kirby, P. Koppel, S. Moore, S. Phillips, D. Maffitt, M. Pringle, L. Tarbox, F. Prior, "The cancer imaging archive (TCIA): Maintaining and operating a public information repository", (2013) 1045-1057. doi:10.1007/s10278-013-9622-7. 


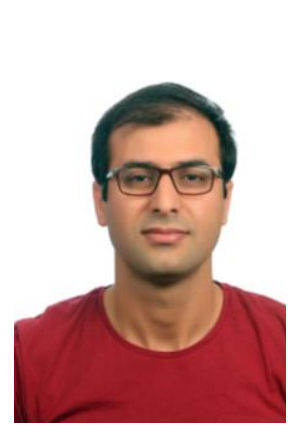

\section{BIOGRAPHY}

Emrah IRMAK was born in 1988 in Mardin, Turkey. He received the B.Sc. degree and M.Sc. degree in Electrical and Electronics Engineering Department from Gaziantep University, Gaziantep, Turkey, in 2012 and 2014, respectively. Emrah Irmak received the Ph.D. degree in Electrical and Electronics Engineering Department from Karabuk University, Karabuk, Turkey in 2018. He studied as a research assistant in Biomedical Engineering Department in Karabuk University between 2012 and 2019. He is currently an Assistant Professor in Electrical and Electronics Engineering Department in Alanya Alaaddin Keykubat University, Antalya, Turkey. His research interests include medical image processing, signal processing and deep learning. 